\title{
Erratum to: Autophagy Reduces Neuronal Damage and Promotes Locomotor Recovery via Inhibition of Apoptosis after Spinal Cord Injury in Rats
}

Peifu Tang • Hongping Hou • Licheng Zhang • Xia Lan •

Zhi Mao • Daohong Liu • Chunqing He • Hailong Du •

Lihai Zhang

Published online: 19 December 2013

(C) Springer Science+Business Media New York 2013

Erratum to: Mol Neurobiol

DOI 10.1007/s12035-013-8518-3

On page 5 and 8 of the article, there were misprints on figures as Figure 3 was published as a duplicate of Figure 2 and at Figure 6, error was found at its imbedded text. The figures are hereby presented and corrected.

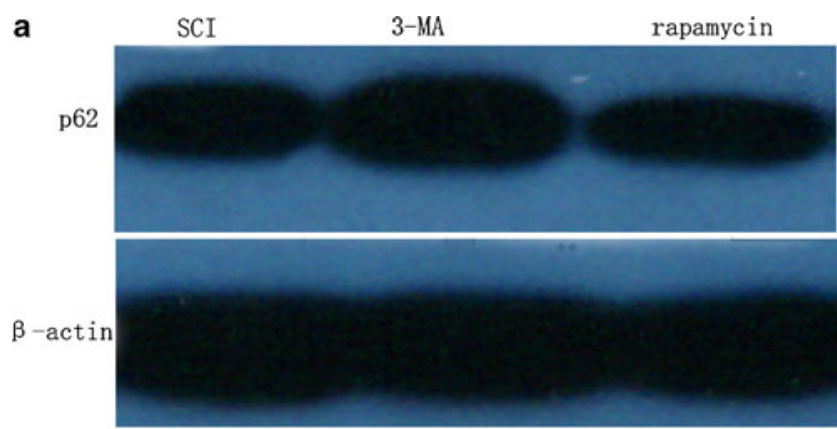

b

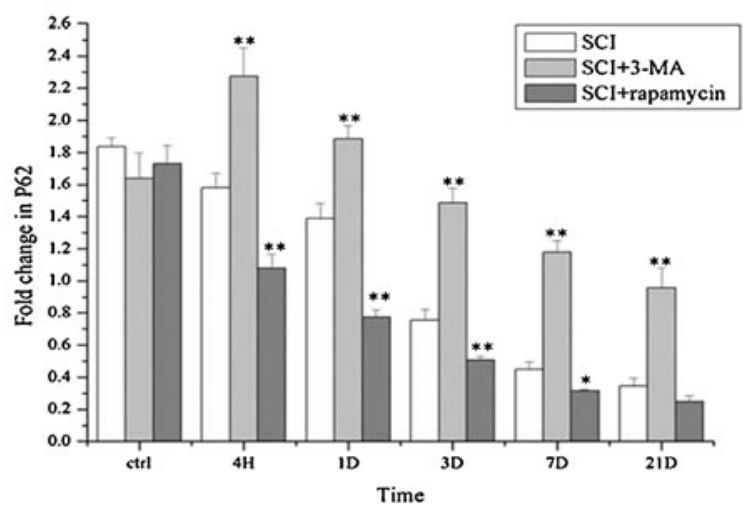

The online version of the original article can be found at http://dx.doi.org/ 10.1007/s12035-013-8518-3.

P. Tang $\cdot$ H. Hou $\cdot$ L. Zhang $\cdot$ X. Lan $\cdot$ Z. Mao $\cdot$ D. Liu $\cdot$ C. He $\cdot$

Fig. 3

H. Du $\cdot$ L. Zhang $(\bowtie)$

Department of Orthopaedics, Chinese PLA General Hospital,

Beijing 100853, People's Republic of China

e-mail: zhanglihai74@yahoo.com.cn

H. Hou

Medical College, Nankai University, No.94 Weijin Road,

Tianjin 300071, People's Republic of China 


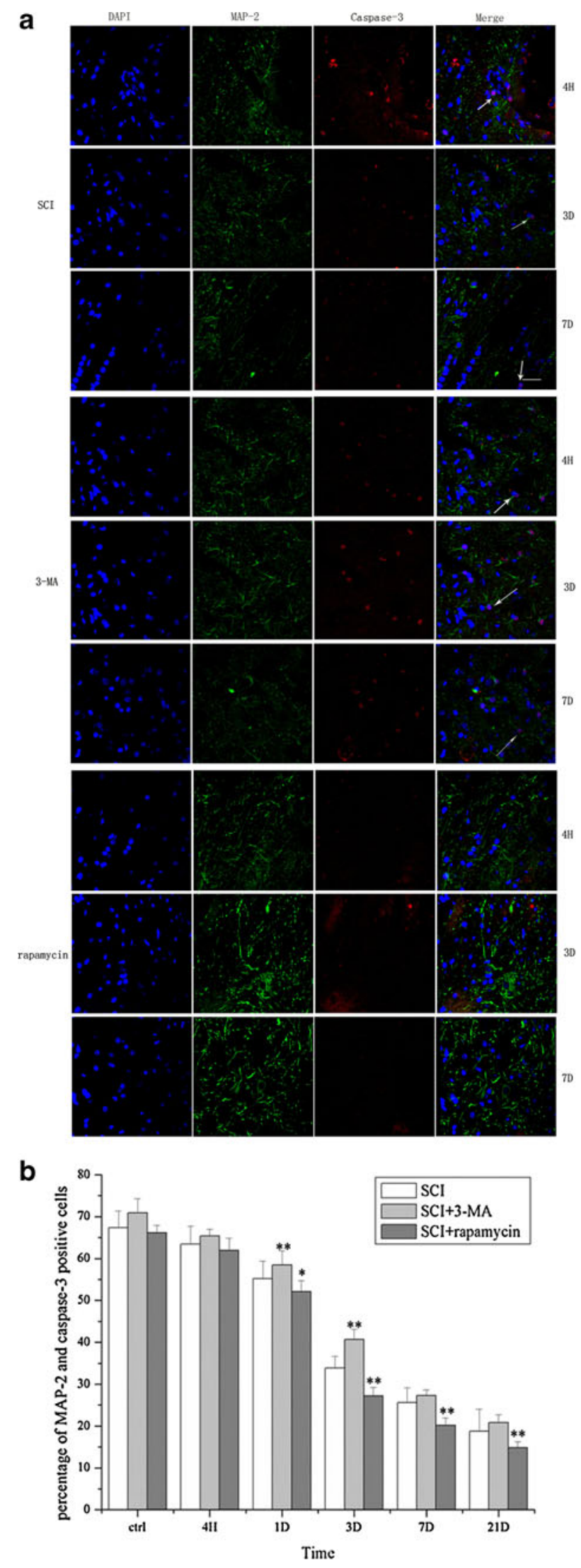

Fig. 6 